\title{
Vibrational Imaging and Quantification of Two-Dimensional Hexagonal Boron Nitride with Stimulated Raman Scattering
}

Jiwei Ling ${ }^{1} \ddagger$ Xianchong Miao ${ }^{1} \ddagger$, Yangye Sun $^{2}$, Yiqing Feng ${ }^{3}$, Liwu Zhang ${ }^{3}$, Zhengzong Sun ${ }^{2}$, Minbiao $\mathrm{Ji}^{{ }^{*}}$

${ }^{1}$ State Key Laboratory of Surface Physics and Department of Physics, Academy for Engineering and Technology, Key Laboratory of Micro and Nano Photonic Structures (Ministry of Education), Fudan University, Shanghai 200433, China

${ }^{2}$ Department of Chemistry and Shanghai Key Laboratory of Molecular Catalysis and Innovative Materials, Fudan University, Shanghai 200433, China

${ }^{3}$ Department of Environmental Science and Engineering, Fundan University, Shanghai 200433, China

\section{Supporting Information}

\section{Variation of the $E_{2 g}$ peak position with pump frequency and delay time.}

As shown in Fig.1d, the SRS peak position of the $E_{2 g}$ mode shifts when the pump frequency changes. This is the direct evidence of Raman signal. In SRS measurements, resonance happens only when the energy difference between pump and Stokes beam matches with phonon energy $(\Omega)$. When the pump frequency changes, which corresponds to the up shift of the orange oval in the figure, the Stokes beam has to move left to maintain the energy difference. Therefore, the resonance time delay changes. 
When the Stokes wavelength is tuned away from the resonance condition, SRS signal decreases as shown in Figure S1a.

The linear relationship between the wavelength and delay time reflects the linear chirp of both beams and can be used to convert time delay into Raman shift. Practically, we also use standard samples with different Raman peaks to calibrate the peak positions.
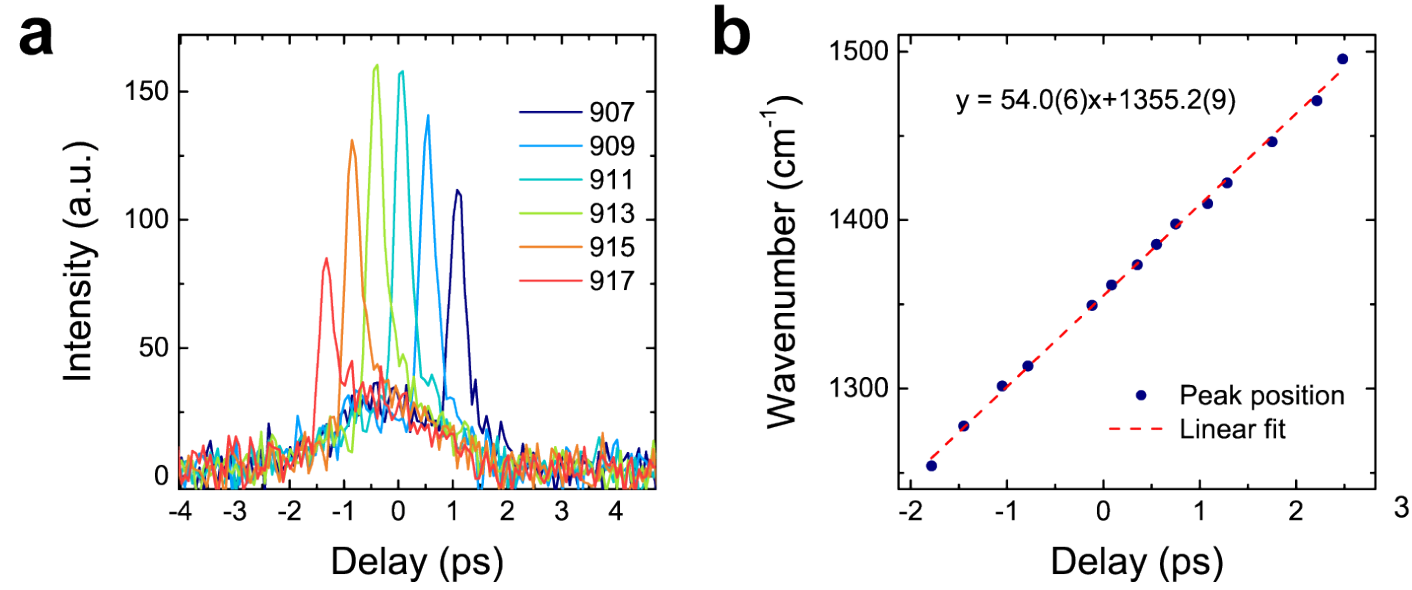

Figure S1. Measured SRS peak intensity under different pump wavelengths and delay times. a) SRS signal under different pump wavelengths (nm). b) The linear relationship between frequency and time can be used to convert time delay to Raman shifts. 


\section{Thickness information of $h-B N$ flakes in the main text.}
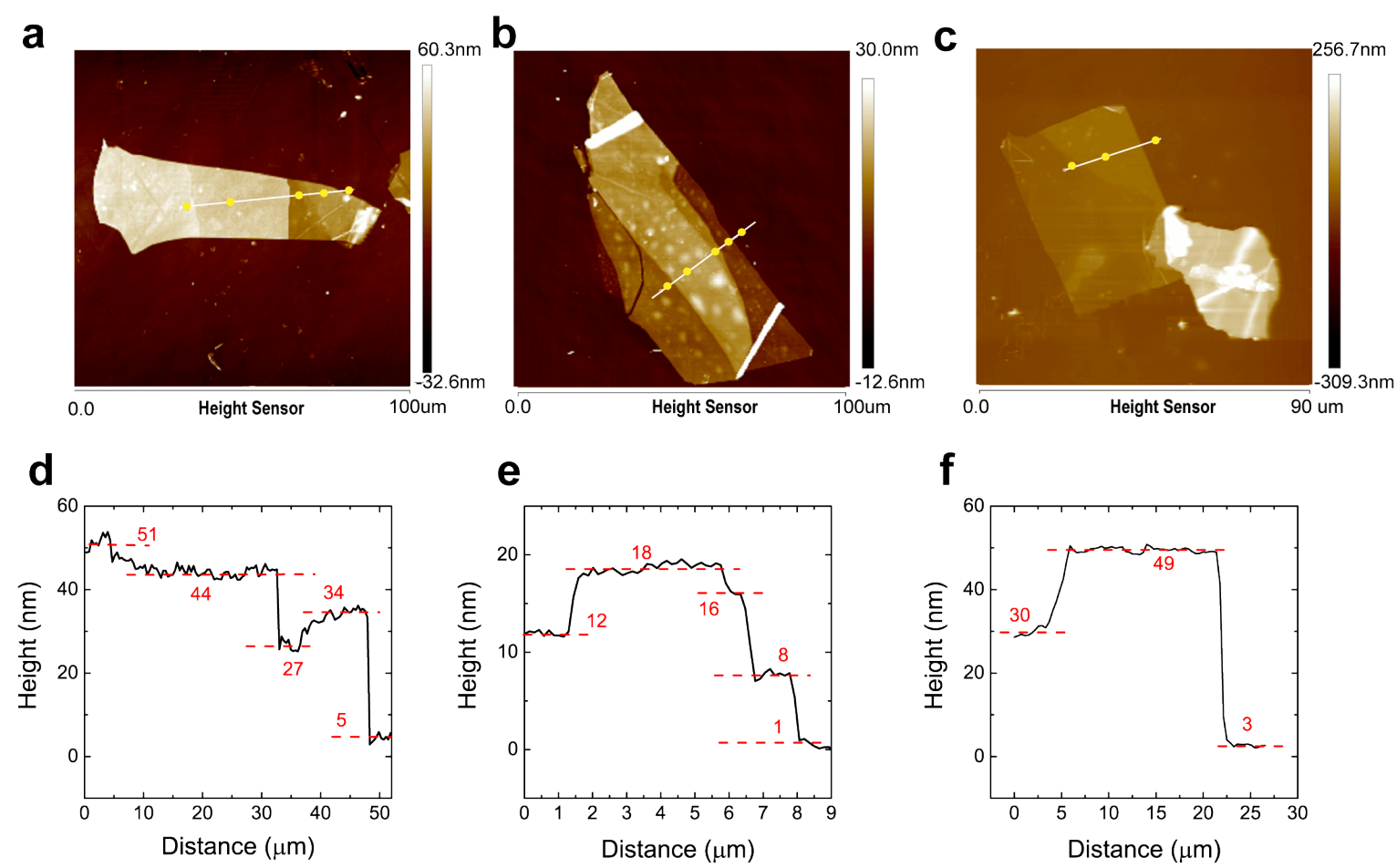

Figure S2. The AFM images of samples in the main text. The AFM images of samples presented in main text Figures 2, 3 and 4 are shown in a), b) and c), respectively. Corresponding line profiles are shown in d)-f). 


\section{Spectral resolution}

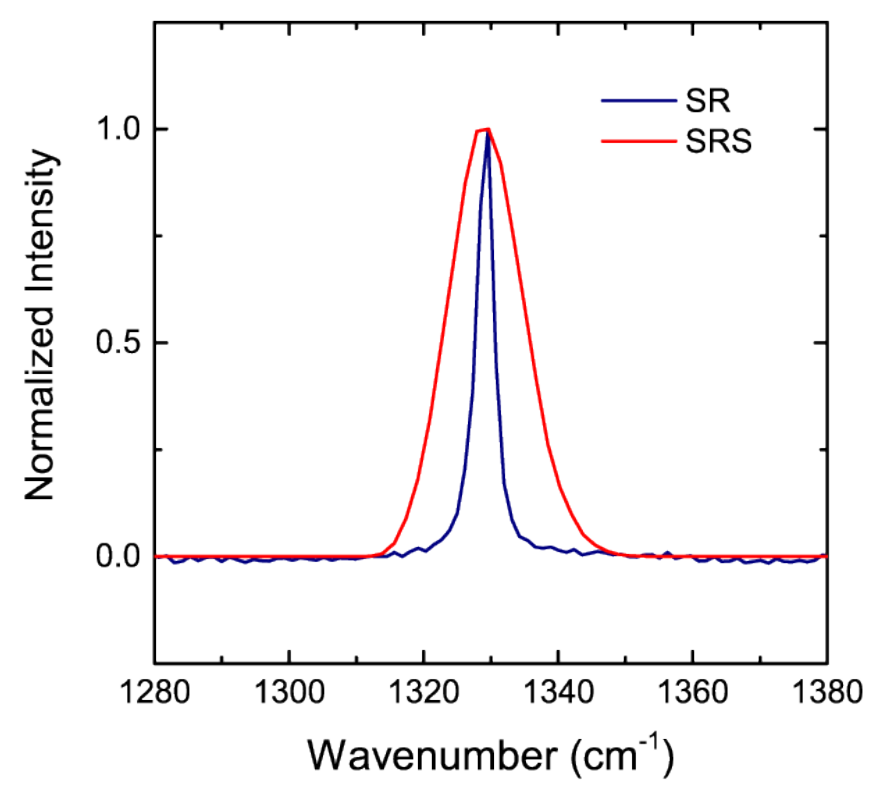

Figure S3. Spectral resolution of SRS system. The spontaneous Raman spectrum and SRS spectrum of diamond microcrystals are measured with diamond. The spontaneous Raman peak of diamond is $\sim 1329 \mathrm{~cm}^{-1}$, and the FWHM is $\sim 2.7 \mathrm{~cm}^{-1}$ (Lorentzian fitting). The red curve shows the SRS spectrum of diamond, with the FWHM of $\sim 12.8 \mathrm{~cm}^{-1}$ (Voigt fitting), which stands for the spectral resolution of our system. 
4. Thicknesses dependent SRS spectra

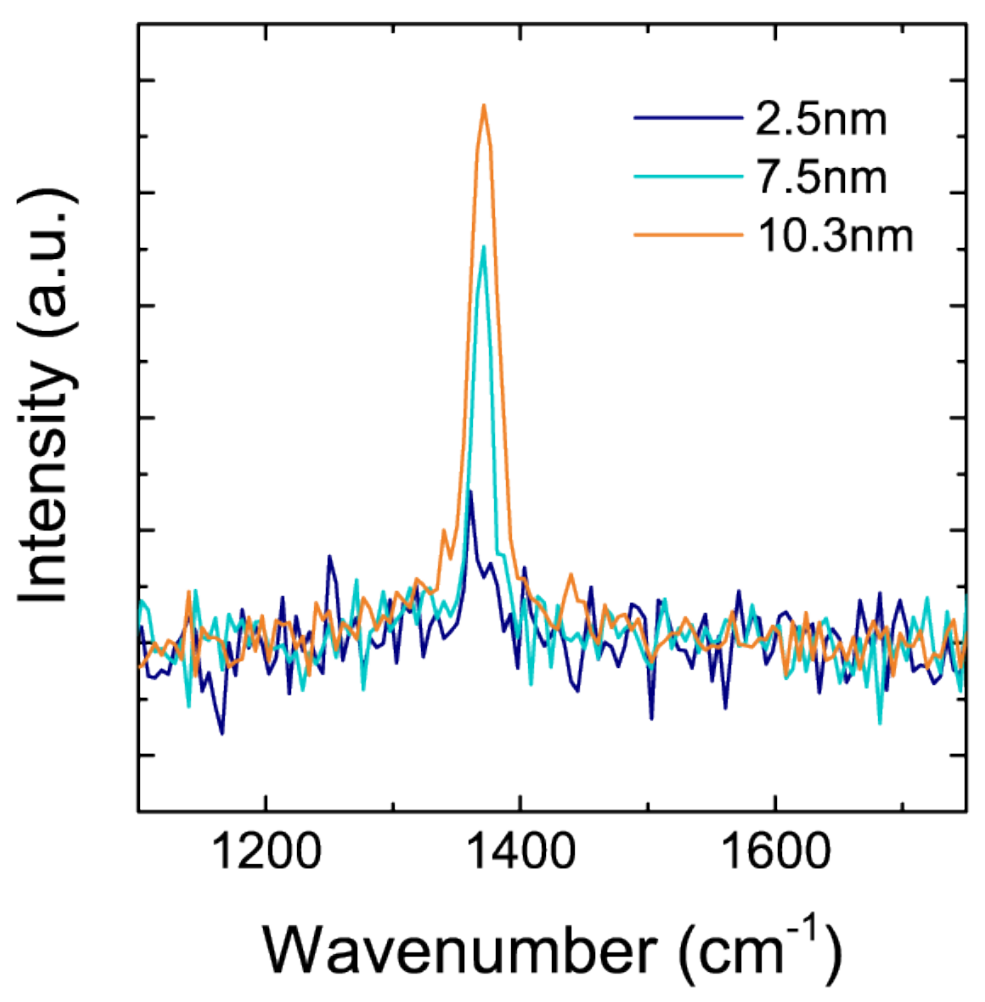

Figure S4. SRS spectra of samples with different thicknesses. SRS spectra of $2.5 \mathrm{~nm}$, $7.5 \mathrm{~nm}$ and $10.3 \mathrm{~nm}$ samples are presented. The spectra were obtained under $913 \mathrm{~nm}$ pump laser, $0.067 \mathrm{ps}$ step, 2 us pixel dwell time without averaging. 


\section{CARS spectrum}

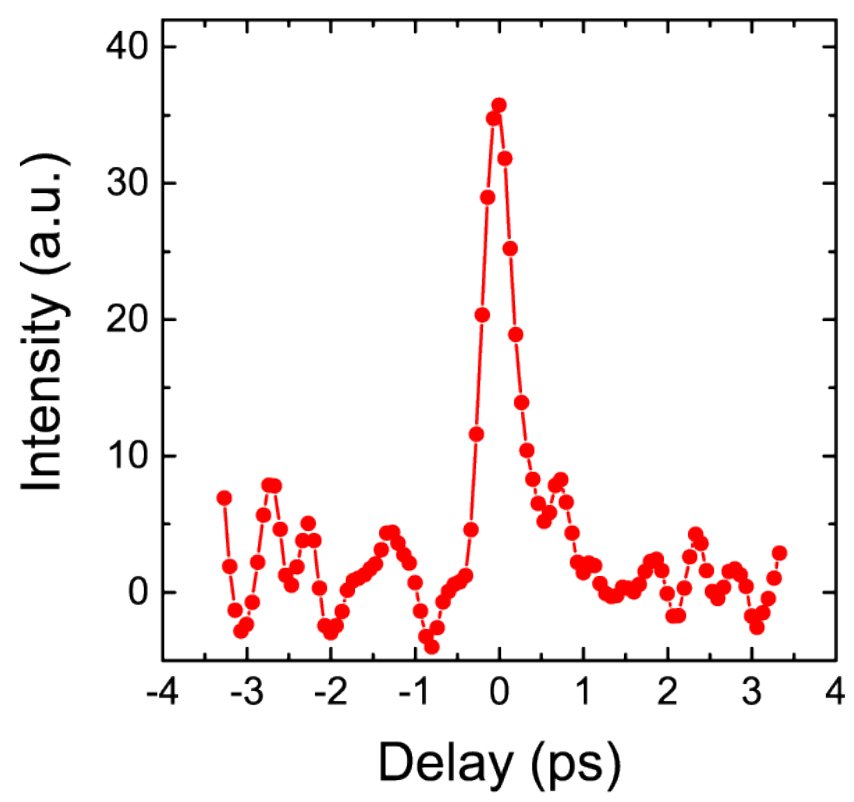

Figure S5. The CARS spectrum of $\mathrm{h}-\mathrm{BN}(\sim \mathbf{1 5 n m})$ with $913 \mathrm{~nm}$ pump laser. The response of the PMT in our set up is low at the CARS wavelength $(\sim 814 \mathrm{~nm})$, thus the CARS spectrum exhibit low signal to noise ratio. However, the resonance feature of CARS spectrum could still be seen with slight distorted line shape, which is well-known because of the non-resonant background. 


\section{Thinnest sample}

a

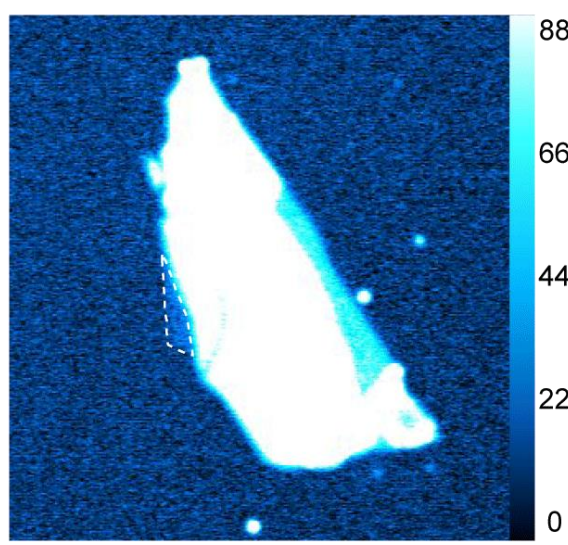

b
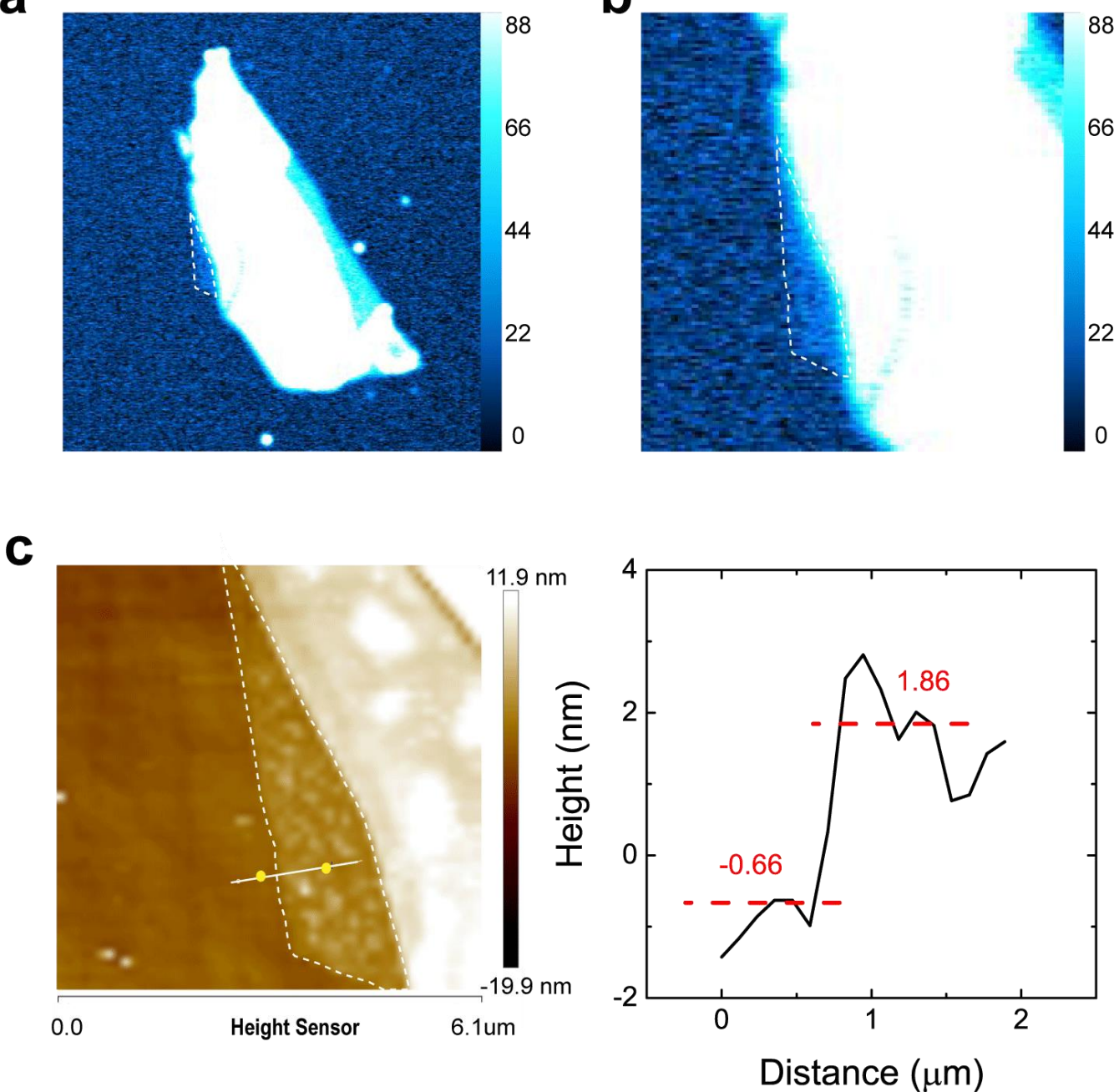

Figure S6. Images of the thinnest sample. a) The region marked by the white dotted boarder is the SRS image of the thinnest h-BN flake we have measured. b) Enlarged image. c) Corresponding AFM image. d) the line profile in c). 


\section{Power dependence}

a

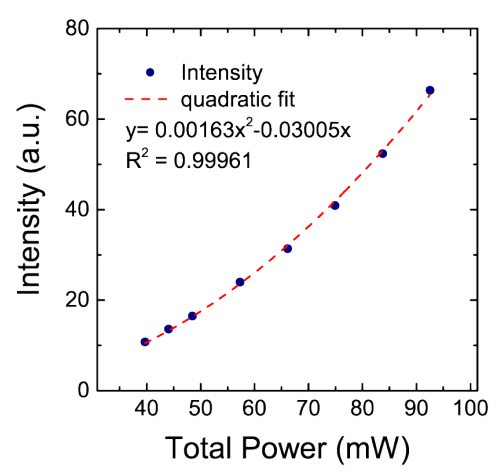

b

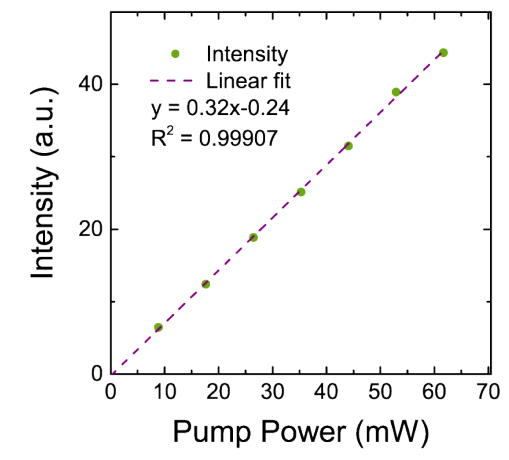

C

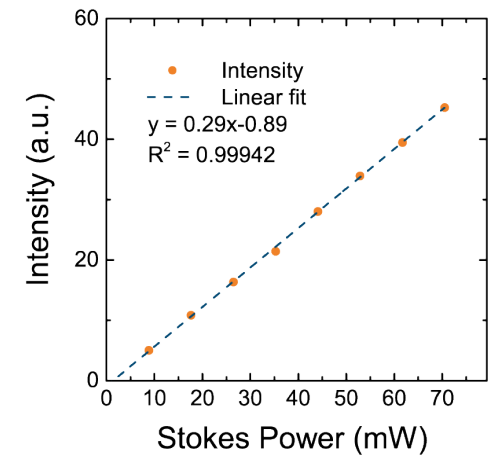

Figure S7. Laser power dependence of SRS intensity. a) SRS intensity under different total power (pump + Stokes) is quadratic. b) When the Stokes power is fixed, the SRS intensity depends linearly on the pump power. c) When pump power is fixed, the SRS signal shows linear dependence on the Stokes power. 


\section{Group velocity dispersion (GVD) calculation}

One way to calculate GVD is using the glass dispersion parameters:

$$
\begin{gathered}
\mathrm{GVD}=\frac{\partial}{\partial \omega}\left(\frac{\partial k}{\partial \omega}\right) \\
\mathrm{k}=\frac{2 \pi n}{\lambda}, \omega=\frac{1}{2 \pi \lambda}
\end{gathered}
$$

So

$$
\mathrm{GVD}=\frac{\lambda^{3}}{2 \pi c^{2}}\left(\frac{\partial^{2} n}{\partial \lambda^{2}}\right)
$$

The reflective index of SF57 glass could be written as Sellmeier formula:

$$
n^{2}-1=\frac{B_{1} \lambda^{2}}{\lambda^{2}-C_{1}}+\frac{B_{2} \lambda^{2}}{\lambda^{2}-C_{2}}+\frac{B_{3} \lambda^{2}}{\lambda^{2}-C_{3}}
$$

Where the parameters could be found in the Schott database:

\begin{tabular}{|l|l|}
\hline $\mathrm{B}_{1}$ & 1.87543831 \\
\hline $\mathrm{B}_{2}$ & 0.37375749 \\
\hline $\mathrm{B}_{3}$ & 2.30001797 \\
\hline $\mathrm{C}_{1}$ & 0.0141749518 \\
\hline $\mathrm{C}_{2}$ & 0.0640509927 \\
\hline $\mathrm{C}_{3}$ & 177.389795 \\
\hline
\end{tabular}

Thus $\frac{\partial^{2} \mathrm{n}}{\partial \lambda^{2}}$ can be calculated from the formula.

Specifically, when $\lambda=910 \mathrm{~nm}, \frac{\partial^{2} n}{\partial \lambda^{2}}=1.34 \times 10^{11}$. Therefore, GVD under $910 \mathrm{~nm}$ is calculated to be $1.792 \times 10^{-25} \mathrm{~s}^{2} / \mathrm{m}$.

On the other hand, the two dimensional mapping shown in Figure 1d, which is similar to FROG can be used to calculate GVD.

The slope of the 2D frequency-time plot in Figure $1 \mathrm{~d}$ is $\alpha=\frac{\partial \varpi}{\partial \tau}=54.1 \mathrm{~cm}^{-1} / \mathrm{ps}$, here 
the frequency (in the unit of wavenumber) is $\varpi=\frac{10^{7}}{\lambda}$, with the wavelength $\lambda$ in the unit of $\mathrm{nm}$.

We can also re-write the slope as: $\alpha=-\frac{10^{7}}{\lambda^{2}} \beta$, where $\beta=\frac{\partial \lambda}{\partial \tau}=-4.48 \mathrm{~nm} / \mathrm{ps}$.

Dispersion parameter of the glass can be represented as:

$$
\begin{gathered}
D=\frac{\partial}{\partial \lambda}\left(\frac{1}{V_{g}}\right)=-\frac{2 \pi c}{\lambda^{2}} G V D \\
D L=\frac{1}{\beta} \\
G V D=-\frac{\lambda^{2}}{2 \pi c L \beta}
\end{gathered}
$$

Where $\mathrm{L}$ is the light path in the glass.

So when $\lambda=910 \mathrm{~nm}, \mathrm{~L}=0.54 \mathrm{~m}$, hence $\mathrm{GVD}=1.818 \times 10^{-25} \mathrm{~s}^{2} / \mathrm{m}$. The value is very close to what we got from the glass parameters.

We can also calculate the pulse width stretched under linear chirp:

For $\lambda=910 \mathrm{~nm}$, calculated GVD $=1.792 \times 10^{-25} \mathrm{~s}^{2} / \mathrm{m}, \mathrm{L}=0.54 \mathrm{~m}$, spectral width of $\Delta \lambda=10 \mathrm{~nm}$, so the pulse width is: $\Delta \tau=\mathrm{DL} \Delta \lambda=2.2 \mathrm{ps}$, which agrees with the measured $2.3 \mathrm{ps}$.

For $\lambda=1040 \mathrm{~nm}$, calculated GVD $=1.94 \times 10^{-25} \mathrm{~s}^{2} / \mathrm{m}, \mathrm{L}=0.66 \mathrm{~m}$, spectral width of $\Delta \lambda=7 \mathrm{~nm}$, so the pulse width is: $\Delta \tau=1.56 \mathrm{ps}$, agrees with the measured $1.5 \mathrm{ps}$. 